\title{
Current and future advances in sirna drug delivery vehicles
}

\begin{abstract}
RNA interference has experienced an exponential increase in research interest since it was first reported nearly 2 decades ago. However, due to difficulties in drug delivery techniques, siRNA-based therapeutics have not reached their fullest potential in the clinical setting. The field of nanotechnology aims at mitigating many of these hurdles through the design and implementation of novel and sophisticated drug delivery vehicles. This review illustrates some of the challenges faced by siRNA drug delivery and considers how new nanomedical approaches can circumvent these biological barriers.
\end{abstract}

Volume 2 Issue 4 - 2015

\author{
Gino KL Delos Reyes, Hicham Fenniri \\ Department of Chemical and Biomedical Engineering, \\ Northeastern University, USA
}

\begin{abstract}
Correspondence: Hicham Fenniri, Department of Chemical and Biomedical Engineering, 3 I 3 Snell Engineering Center 360 Huntington Avenue, Northeastern University, Boston, MA 02II5, USA, Tel (6I7) 373-7690 Email h.fenniri@nen.edu
\end{abstract}

Received: April 17, 2015 | Published: September 14, 2015
Abbreviations:RNAi, RNA Interference; siRNA, Small Interfering RNA; dsRNA, Double Stranded RNA; RISC, RNAInduced Silencing Complex; mRNA, Messenger RNA; TLR, TollLike Receptor

\section{Introduction}

Ever since Fire \& Mello's. ${ }^{1}$ seminal paper on the discovery of the RNA interference (RNAi) pathway in 1998, a panoply of academic and industrial groups alike have devoted their research efforts to take advantage of the gene-knockdown capabilities of small interfering RNA (siRNA). ${ }^{2-4}$ siRNA has proven to be an efficient means of silencing a specific gene of interest and thus has led to the exponential increase in research publications regarding therapeutic approaches utilizing siRNA. ${ }^{5}$ Because of its simplicity and elegance, synthetic siRNA transfection has become an attractive and powerful tool for silencing specific phenotypes. ${ }^{6}$ All that is needed to knock down a gene is the transfection of a short double stranded RNA (dsRNA) into the cell. Once in the cytoplasm, this double stranded siRNA is cleaved by a protein called Dicer and subsequently conjugated to the RNAinduced silencing complex (RISC). The RISC complex then seeks out a complementary messenger RNA (mRNA) molecule and degrades it, thus inhibiting the expression of the target phenotype. ${ }^{1,7}$ Because of the specificity of gene targeting and the ease of transfection of siRNA into cells, RNA interference has shown much promise in disease therapeutics. ${ }^{8}$ Theoretically, if the siRNA molecule is designed appropriately, the RNAi pathway can be exploited to target the silencing of virtually any gene in the body, allowing for a broad therapeutic potential.

Despite all of the potential surrounding siRNA-based therapeutics, a myriad of challenges need to be addressed in order for RNAi to exhibit success in the clinical setting. ${ }^{9}$ The most important hurdle that needs to be overcome is the potential for "off-target" effects. A gene that shares a strong homology to the target gene has the potential to be inadvertently knocked down, leading to possibly severe unintended side effects. Another barrier to drug delivery and siRNA therapy is immune stimulation. Various groups have shown that delivery of naked synthetic siRNA is a strong activator of the toll-like receptor (TLR) pathway, thus leading to a robust innate inflammatory immune response. ${ }^{10}$ Lastly, the delivery of the siRNA itself has proven to be a challenge to many researchers. Upon administration in the bloodstream, naked siRNA face a multitude of serum nucleases whose main purpose is to seek out and degrade foreign nucleic acids. ${ }^{11}$ Nucleases, alongside a relatively swift renal clearance rate, drastically shorten the siRNA circulation time in the blood stream. Once the siRNA reaches the organ or cells of interest, it must then diffuse through a dense extracellular matrix composed of networks of polysaccharides and fibrous proteins. ${ }^{12}$ Moreover, if the siRNA manages to successfully diffuse, it must then become engulfed by the cell through receptor mediated endocytosis and escape from the endosomal compartment in order to show any therapeutic ability. ${ }^{12}$ These challenges in drug delivery and immune protection need to be overcome to enable siRNA therapies to make it past the clinic. However, through the use of nanotechnology, many of these issues cannot only be addressed, but also mitigated. ${ }^{3}$

The field of nanotechnology has opened the door to a plethora of efficacious and innovative drug delivery systems.${ }^{13}$ Many labs have presented solutions to siRNA delivery utilizing novel nanotechnologybased carrier systems. One potentially promising and novel method for efficacious delivery of therapeutics such as siRNA is through Rosette Nanotubes (RNTs). First reported by our group in 2001, RNTs are a novel class of supra molecular self-assembled nanotubes that have shown efficacy in delivering a multitude of therapeutics. ${ }^{14}$ The building blocks of the RNTs are fused guanine-cytosine bases, hereby referred to as $\mathrm{G} \wedge \mathrm{C}$ bases that entropically form a six membered rosette through Watson-Crick hydrogen bonding. These rosettes then stack upon one another through pi bonding, forming rosettes of tunable length.${ }^{15}$ The major advantage to the RNT system is that the G^C building blocks can be functionalized with a variety of functional groups such as aptamers, proteins or peptides. These functional groups are then displayed simultaneously on the surface of the RNT, allowing for the attachment of different functionalities such as cellular targeting and immune evasion. ${ }^{16}$ Based on unpublished preliminary data, RNTs that display positively charged functional groups such as poly-lysine are able to form tight bonds through electrostatic interactions with siRNA, while also sterically hindering exonuclease and endonuclease activity.

Liposome-like drug delivery vehicles, termed "lipidoid" nanoparticles by the Anderson group, have also shown high efficacy in siRNA delivery. ${ }^{17} \mathrm{~A}$ large library of these lipidoid nanoparticles are synthesized through the combination of various alkyl-acrylates and amines, which form a liposome-like structure in solution. By screening this library for the ability to complex siRNA and enter 
the target cell, they were able to identify efficacious siRNA delivery systems. The payload siRNA molecule can thus be encapsulated in the positively charged core of the lipidoid, essentially protecting it from serum degradation and off-target immunological effects. ${ }^{18}$ The surfaces of the lipidoid can then be targeted to certain cells through a variety of different moieties, enabling endocytosis and endosomal escape. Using their system, they were able to observe a remarkable gene silencing ability of $65 \%$ in their in vivo mouse model. ${ }^{19}$ Their work shows much promise for the future of siRNA delivery.

The RNA interference pathway provides a multitude of advantages over traditional pharmaceutical drugs for the treatment of various diseases. Moving forward, we expect that more research groups will harness the power of nanotechnology to improve upon the status quo of siRNA delivery. ${ }^{20}$ Although substantial progress has been made in the field of in vivo siRNA delivery, the research is still in a relatively nascent stage and there are many improvements that have to be made and many challenges that have yet to be overcome. ${ }^{8}$ For example, better strategies to mitigate off-target effects and undesirable immunological stimulation need to be addressed. ${ }^{21}$ Furthermore, the pharmacokinetic and pharmacodynamic data have yet to be well elucidated in many of the in vivo studies performed. ${ }^{22}$

\section{Conclusion}

To conclude, the field of siRNA therapeutics has made significant advancements since Fire \& Melo first demonstrated gene knockdown in C. elegans.$^{1,10}$ Delivery remains the most significant barrier to siRNA therapeutics having successful outcomes in the clinical settings. ${ }^{11}$ Research must remain focused on identifying the specific biological phenomena and mechanisms through the use of various nano-based platforms in order to elucidate proper guidelines for siRNA delivery vehicles. Creativity and innovation are necessary for the advancement of nanotechnology approach to RNA interference.

\section{Acknowledgments}

None.

\section{Conflicts of interest}

None.

\section{References}

1. Fire $\mathrm{A}, \mathrm{Mello} \mathrm{C}, \mathrm{Xu} \mathrm{S}$ et al. Potent and specific genetic interference by double-stranded RNA in Caenorhabditis elegans. Nature. 1998;391(6669):806-811

2. Tuschl T RNA interference and small interfering RNAs. Chembiochem 2001;2(4):239-245.

3. Nakamura Y, Kogure K, Futaki S Octaarginine-modified multifunctional envelope-type nano device for siRNA. $J$ Control Release. 2007;119(3):360-367.
4. Nguyen J, Steele TWJ, Merkel O et al. Fast degrading polyesters as siRNA nano-carriers for pulmonary gene therapy. J Control Release. 2008;132(3):243-251.

5. Lytton Jean AKR, Langer R, Anderson DG Five years of siRNA delivery: Spotlight on gold nanoparticles. Small. 2011;7(14):1932-1937.

6. Vickers TA, Koo S, Bennett CF et al. Efficient reduction of target RNAs by small interfering RNA and RNase $\mathrm{H}$-dependent antisense agents. A comparative analysis. J Biol Chem. 2003;278(9):7108-7118.

7. Daka A, Peer D (2012) RNAi-based nanomedicines for targeted personalized therapy. Adv Drug Deliv Rev. 2003;64(13):1508-1521.

8. Oh YK, Park TG siRNA delivery systems for cancer treatment. $A d v$ Drug Deliv Rev. 2009;61(10):850-862.

9. Pecot CV, Calin GA, Coleman RL et al. RNA interference in the clinic: challenges and future directions. Nat Rev Cancer. 2011;11(1):59-67.

10. Stallwood Y RNA interference. Pharmacogenomics. 2005;6(1):13-16.

11. Layzer JM, McCaffrey AP, Tanner AK et al. In vivo activity of nucleaseresistant siRNAs. RNA. 2004;10(5):766-771.

12. Medarova $\mathrm{Z}$, Pham $\mathrm{W}$, Farrar $\mathrm{C}$ et al. In vivo imaging of siRNA delivery and silencing in tumors. Nat Med 2007;13(3):372-377.

13. Sahay G, Alakhova DY, Kabanov AV Endocytosis of nanomedicines. $J$ Control Release. 2010;145(3):182-195.

14. Fenniri H, Mathivanan P, Vidale KL et al. Helical rosette nanotubes: Design, self-assembly, and characterization. $J$ Am Chem Soc. 2001;123(16):3854-3855

15. Fenniri H, Deng BL, Ribbe AE et al. Entropically driven self-assembly of multichannel rosette nanotubes. Proc Natl Acad Sci. 2002;99(Suppl 2):6487-6492.

16. Zhang L, Webster TJ Nanotechnology and nanomaterials: Promises for improved tissue regeneration. Nano Today. 2009;4(1):66-80.

17. Whitehead KA, Dorkin JR, Vegas AJ et al. Degradable lipid nanoparticles with predictable in vivo siRNA delivery activity. Nat Commun. 2014;5:4277.

18. Kanasty RL, Whitehead KA, Vegas AJ et al. Action and Reaction: The Biological Response to siRNA and Its Delivery Vehicles. Mol Ther 2012;20(3):513-524.

19. Love KT, Mahon KP, Levins CG et al. Lipid-like materials for lowdose, in vivo gene silencing. Proc Natl Acad Sci. 2010;107(5):1864-1869.

20. Lin $\mathrm{G}, \mathrm{Hu} \mathrm{R}$, Law WC et al. Biodegradable nanocapsules as siRNA carriers for mutant $\mathrm{k}$-ras gene silencing of human pancreatic carcinoma cells. Small. 2013;9(16):2757-2763.

21. Varkouhi AK, Scholte M, Storm G, Haisma HJ (2011) Endosomal escape pathways for delivery of biologicals. J Control Release 151(3): $220-228$.

22. Wang J, Byrne JD, Napier ME, DeSimone JM (2011) More effective nanomedicines through particle design. Small 7(14): 1919-1931. 